TOKYO J. Math.

Vol. 7 , No. 1,1984

\title{
On Peak Sets for the Real Part of a Function Space
}

\author{
Shinya YAMAGUCHI and Junzo WADA
}

Waseda University

\section{Introduction.}

Let $A$ be a function space on a compact Hausdorff space $X$. In this paper, we give conditions for certain families of closed subsets in $X$ under which $A$ is characterized. In particular, in $\S 2$ we show that if any peak set for the real part of $A$ is a BEP-set for $A$, then $A=C(X)$ (Theorem 2.2). A theorem in the case of function algebras corresponding to it has obtained by Briem [4].

Throughout this paper, $X$ will denote a compact Hausdorff space. $A$ is said to be a function space (resp. function algebra) on $X$ if $A$ is a closed subspace (resp. subalgebra) in $C(X)$ containing constant functions and separating points in $X$. Let $A$ be a function space and $F$ be a closed subset in $X$. We say that $A \mid F$ has the norm preserving extension property if for any $f \in A$ there is a $g \in A$ such that $g=f$ on $F$ and $\|g\|=\|f\|_{F}$, where $\|g\|=\sup _{x \in X}|g(x)|$ and $\|f\|_{F}=\sup _{x \in F}|f(x)|$. Such a closed subset $F$ is called an NPEP-set for $A$. For a closed subset $F$ in $X$, we put $\hat{F}=\left\{x \in X:|f(x)| \leqq\|f\|_{F}\right\}$ for any $f \in A$.

Let $A$ be a function space on $X . A^{\perp}$ denotes the measures $\mu$ on $X$ such that $\int f d \mu=0$ for any $f \in A$, and $E$ denotes the closure of $\cup_{\mu \in A^{\perp}} \operatorname{supp} \mu . \quad E$ is the smallest one in the family of closed subsets $F$ in $X$ which satisfy the following property: $f \in A$ whenever $f \in C(X)$ and $f(x)=0$ for any $x \in F$. We call $E$ the essential set for $A$ (see [8] for essential sets in the case of function algebras).

Let $A$ be a function algebra on $X$. The following is due to Glicksberg [7]: If $A \mid F$ is closed in $C(F)$ for any closed subset $F$ in $X$, then $A=$ $C(X)$. In the case of function spaces $A$, Briem [3] has shown that $A=$ $C(X)$ if any closed subset $F$ in $X$ is any NPEP-set for $A$.

On the other hand, when $A$ is a function algebra on $X$, Briem [4] has given conditions for peak sets for the real part $\operatorname{Re} A$ of $A$ under which $A$ coincides with $C(X)$.

Received March 30, 1983 
In this paper, we give, in $\S 1$, a slight extension of the theorem of Briem for NPEP-sets stated above. In $\S 2$, we give a result on peak sets for $\operatorname{Re} A$ in the case of function spaces in association with the theorem of Briem for peak sets for $\operatorname{Re} A$ in the case where $A$ is a function algebras.

\section{§ 1. NPEP-sets.}

The following lemma is obtained by a similar way as in the proof of Briem ([3], Theorem 2).

Lemma 1.1. Let $A$ be a function space and $F_{0}$ be a closed subset in $X$. If any closed subset $F$ containing $F_{0}$ is an NPEP-set for $A$, then $\hat{F}_{0} \supset \cup_{\mu \in A^{\perp}}$ supp $\mu$, and hence $\hat{F}_{0} \supset E$.

Proof. We first have that for any $x, y \in X \backslash \hat{F}_{0}, x \neq y$, there is an $a \in A$ such that $a \mid\left(\hat{F}_{0} \cup\{y\}\right)=0$ and $a(x)=1$. For otherwise, $a_{1}(x)=a_{2}(x)$, whenever $a_{1}=a_{2}$ on $\hat{F}_{0} \cup\{y\}, a_{1}, a_{2} \in A$. Hence the mapping $\varphi: a \mid \hat{F}_{0} \cup\{y\} \rightarrow$ $a(x)$ is a well-defined linear functional on $A \mid\left(\hat{F}_{0} \cup\{y\}\right)$. Since $\hat{F}_{0} \cup\{y\}$ is an NPEP-set for $A$, the norm of $\varphi$ is 1 . Hence $a(x)=\int_{\hat{F}_{0} \cup\{y \mid} a d \mu(a \in A)$ for a measure $\mu$ on $\hat{F}_{0} \cup\{y\}$ with $\|\mu\|=1$. We easily see that $|\mu|\left(\hat{F}_{0}\right)>0$. Since $x \notin \hat{F}_{0}, 1=a_{0}(x)>\left\|a_{0}\right\| \hat{F}_{0}$ for an $a_{0} \in A$. We can assume that $\left|a_{0}(y)\right| \leqq 1$ since $\hat{F}_{0} \cup\{x\}$ is an NPEP-set for $A$.

$$
1=a_{0}(x)=\int_{\hat{F}_{0} \cup\{y\}} a_{0} d \mu \leqq\left\|a_{0}\right\|_{\hat{F}_{0}}|\mu|\left(\hat{F}_{0}\right)+|\mu|(\{y\})<|\mu|\left(\hat{F}_{0}\right)+|\mu|(\{y\})=\|\mu\|=1 .
$$

From this contradiction it follows that $a \mid\left(\hat{F}_{0} \cup\{y\}\right)=0, a(x)=1$ for an $a \in A$.

Similarly, we can show that for any closed subset $F \supset \hat{F}_{0}$ and for any $x \in X \backslash F$, there is an $a \in A$ with $a \mid F=0$ and $a(x)=1$. From this, we have that for any closed subset $F \supset \widehat{F}_{0}$ and any closed subset $G$ with $F \cap G=\varnothing$, there is an $a \in A$ such that $a|F=0, \operatorname{Re} a| G>0$ and $a(X) \subset D=$ $\{z \in C:|z-1 / 2| \leqq 1 / 2\}$. We put $L(F, G)=\sup \{r \in R: \operatorname{Re} a \mid G \geqq r$ for an $a \in A$ with $a \mid F=0, a(X) \subset D$ \}. By a similar manner as in the proof of Briem ([3], Lemma 6), we have that $L(F, G)>2^{-11}$ for any closed subset $F \supset \hat{F}_{\mathrm{f}}$ and any closed subset $G$ with $F \cap G=\varnothing$. This implies that supp $\mu \subset \hat{F}_{0}$ for any $\mu \in A^{\perp}$. For, if $|\mu|\left(X \backslash \hat{F}_{0}\right)>0$ for a $\mu \in A^{\perp}$, we can assume that $|\mu|\left(X \backslash \widehat{F}_{0}\right)=1$.

Here we can find finitely many mutually disjoint closed subsets $F_{1}, F_{2}, \cdots, F_{m} \subset X \backslash \hat{F}_{0}$ and $z_{i} \in C,\left|z_{i}\right|=1 \quad(i=1,2, \cdots, m)$ such that the measure $\lambda=z_{1}|\mu|_{F_{1}}+\cdots+z_{m}|\mu|_{F_{m}}$ satisfies that $\left\|\lambda-\mu_{X \backslash \hat{F}_{0}}\right\|<2^{-12}$. Put $\nu=$ $\mu_{F_{0}}+\lambda$. Then $\|\nu-\mu\|<2^{-12}$. From the fact above, there are $b_{i} \in A$ such 
that $b_{i}\left|\left(\hat{F}_{0} \cup F_{j}\right)=0(j \neq i), \operatorname{Re} b_{i}\right| F_{i} \geqq 2^{-11}$ and $b_{i}(X) \subset D(i=1,2, \cdots, m, j=$ $1,2, \cdots, m)$. If we set $a=\left(\bar{z}_{1} b_{1}+\cdots+\bar{z}_{m} b_{m}\right) \mid\left(\hat{F}_{0} \cup F\right), \quad F=\cup_{i=1}^{m} F_{i}$, then $a \in A \mid\left(\hat{F}_{0} \cup F\right),\|a\|_{\hat{F}_{0} \cup F} \leqq 1$. Hence $c \mid \hat{F}_{0} \cup F=a,\|c\| \leqq 1$ for a $c \in A$. An easy calculation shows that $\operatorname{Re} \int c d \nu \geqq 2^{-11}\left(1-2^{-12}\right)$. On the other hand, $\operatorname{Re} \int c d \nu \leqq\left|\int c d \nu\right|=\left|\int c d \nu-\int c d \mu\right| \leqq\|\nu-\mu\|<2^{-12}$ since $c \in A$ and $\mu \in A^{\perp}$. This contradiction proves the lemma.

From Lemma 1.1, we have

THEOREM 1.2. Let $A$ be a function space on $X$ and $F_{0}$ be a closed subset in $X$. If $F$ is an NPEP-set for $A$ for any closed subset $F$ containing $F_{0}$, then $F_{0} \supset \partial_{A \mid E}$, where $\partial_{A \mid E}$ denotes the Shilov boundary for the function space $A \mid E$.

Proof. We first show that $\hat{F}_{0}=F_{0} \cup E$. From Lemma 1.1, we have $\hat{F}_{0} \supset F_{0} \cup E$. If $x \notin F_{0} \cup E$, there is an $a \in C(X)$ such that $a \mid\left(F_{0} \cup E\right)=0$, $a(x)=1,0 \leqq a \leqq 1$ and $a \mid E=0$. It implies that $a \in A$ and $a(x)>\|a\|_{F_{0}}$. It shows that $x \notin \hat{F}_{0}$ and $\hat{F}_{0}=F_{0} \cup E$. Let $\left\{G_{\alpha}: \alpha \in I\right\}$ be the family of closed neighborhoods of $E$. Then $E=\cap_{\alpha \in I} G_{\alpha}$. Let $a \in A$. Suppose that $G_{\alpha_{0}} \cap$ $\left\{x \in F_{0}:|a(x)| \geqq\|a\|_{E}\right\}=\varnothing$ for an $\alpha_{0} \in I$. Then there is an $h \in C(X)$ such that $0 \leqq h \leqq 1, h(E)=1$ and $h\left(X \backslash G_{\alpha_{0}}\right)=0$.

Now, since $h a=a$ on $E$, we have that $h a \in A$, and

$$
\begin{array}{cl}
|h a(x)| \leqq|a(x)|<\|a\|_{E}=\|h a\|_{E} & \text { if } x \in F_{0} \cap G_{\alpha_{0}}, \\
h a(x)=0 & \text { if } x \in F_{0} \mid G_{\alpha_{0}} .
\end{array}
$$

It follows that $\|h a\|_{E}>\|h a\|_{F_{0}}$ and it is a contradiction since $\hat{F}_{0} \supset E$. From this, for any $\alpha \in I, \quad G_{\alpha} \cap\left\{x \in F_{0}:|a(x)| \geqq\|a\|_{E}\right\} \neq \varnothing$. That is, $\left\{G_{\alpha} \cap\left\{x \in F_{0}:|a(x)| \geqq\|a\|_{E}\right\}\right\}$ has the finite intersection property and hence $E \cap\left\{x \in F_{0}:|a(x)| \geqq\|a\|_{E}\right\} \neq \varnothing$. Thus $\partial_{A \mid E} \subset E \cap F_{0} \subset F_{0}$.

CoRollary 1.3. Let $A$ be a function space and $F_{1}, F_{2}$ be two closed subsets in $X$ with $F_{0} \cap F_{1}=\varnothing$. If any closed subset containing $F_{0}$ is an NPEP-set for $A$ and any closed subset containing $F_{1}$ is an NPEP-set for $A$, then $A=C(X)$.

Corollary 1.4 (Briem [3]). Let $A$ be a function space. If any closed subset in $X$ is an NPEP-set for $A$, then $A=C(X)$.

REMARK. In the case of function algebras, the following fact corresponds to Theorem 1.2: Let $A$ be a function algebra on $X$ and $F_{0}$ be a closed subset in $X$. If $A \mid F$ is closed in $C(F)$ for any closed subset $F$ 
containing $F_{0}$, then $F_{0} \supset \partial_{A \mid E}$ (cf. [9]).

\section{§ 2. Peak sets for $\operatorname{Re} A$.}

We here give conditions for peak sets for $\operatorname{Re} A$ under which $A$ coincides with $C(X)$ in the case where $A$ is a function space.

When $A$ is a function space, the following properties on a closed set $F$ in $X$ are equivalent (cf. [6]): (1) $\mu \in A^{\perp}$ implies $\mu_{F} \in A^{\perp}$. (2) $A$ has the bounded extension property with respect to $F$, i.e., for every $f \in A \mid F$ and each closed set $G$ in $X$ with $G \cap F=\varnothing$, and for each $\varepsilon>0$, there exists a $g \in A$ such that $g \mid F=f,\|g\|=\|f\|_{F}$ and $\|g\|_{G}<\varepsilon$. Such a subset $F$ is called a $B E P$-set for $A$.

Lemma 2.1. Let $A$ be a function space. If any peak set for $\operatorname{Re} A$ is a BEP-set for $A$, then $A$ is self-adjoint, that is, if $f \in A$, then $\bar{f} \in A$.

Proof. It is sufficient to show that if a peak set $F$ for $\operatorname{Re} A$ is a BEP-set for $A$, then $F$ is an $M$-hull (cf. [1], Theorem 9.1, p. 220). In order to prove that $F$ is an $M$-hull, we need to show that $F=\mathscr{F} \cap X$ for some closed face $\mathscr{F}$ in $S_{A}=\left\{L \in A^{*}: L(1)=1=\|L\|\right\}$ (cf. [1], Proposition 2.7, p. 158). We can construct $\mathscr{F}$ satisfying the above as follows: $\mathscr{F}=\left\{L \in A^{*}: L(f)=\int f d \mu(f \in A)\right.$ for a measure $\mu$ such that $\mu \geqq 0,\|\mu\|=1$ and supp $\mu \subset F\}$. If $\mathscr{F} \in L=\left(L_{1}+L_{2}\right) / 2, L_{1}, L_{2} \in S_{A}$, then $L_{i}(f)=\int f d \mu_{i}$ $(f \in A)$ with some measure $\mu_{i} \geqq 0\left\|\mu_{i}\right\|=1(i=1,2)$. Since $\mu-\left(\mu_{1}+\mu_{2}\right) / 2 \epsilon$ $A^{\perp}$ and $F$ is a BEP-set for $A, \mu_{F}-\left\{\left(\mu_{1}\right)_{F}+\left(\mu_{2}\right)_{F}\right\} / 2 \in A^{\perp}$. We here have $\mu=\mu_{F}$. It implies that $\left(\mu_{1}\right)_{X \backslash F}+\left(\mu_{2}\right)_{X \backslash F} \in A^{\perp}$ and hence $\int_{X \backslash F} d \mu_{1}+\int_{X \backslash F} d \mu_{2}=0$. So $\mu_{1}(X \backslash F)=\mu_{2}(X \backslash F)=0$ and $L_{1}, L_{2} \in \mathscr{F}$. This shows that $\mathscr{F}$ is a face of $S_{\Delta}$. To show that $F=\mathscr{F} \cap X$, let $L_{x} \in \mathscr{F} \cap X\left(L_{x}(f)=f(x), f \in A\right)$. Then $L_{x} \in \mathscr{F}$, and $x \in F$ since $F$ is a peak set for $\operatorname{Re} A$, and this proves the lemma.

In association with a theorem of Briem ([4], Theorem 3), we can obtain the following in the case of function spaces.

THEOREM 2.2. Let $A$ be a function space. Then the following four properties are equivalent.

(i) Any closed subset in $X$ containing a peak set for $\operatorname{Re} A$ is an NPEP-set for $A$.

(ii) Any peak set $F_{0}$ for $\operatorname{Re} A$ is a BEP-set for $A$.

(iii) $A=C(X)$.

(iv) Any closed subset in $X$ is an NPEP-set for $A$. 
REMARK. When $A$ is a function algebra, the property (ii) above is equivalent to (ii) in Theorem 3 of Briem ([4]).

Proof. (i) $\rightarrow$ (ii). Let $F_{0}$ be a peak set for $\operatorname{Re} A$. By (i) and Lemma 1.1, $\hat{F}_{0} \supset \cup_{\mu \in A^{\perp}}$ supp $\mu$. On the other hand, $\hat{F}_{0}=F_{0}$ since $F_{0}$ is a peak set for $\operatorname{Re} A$. For, if $x_{0} \in \hat{F}_{0} \backslash F_{0}$, we put $L\left(a \mid F_{0}\right)=a\left(x_{0}\right)$ for $a \in A$. Then $L$ is well-defined and it is a linear functional on $A \mid F_{0}$ with $\|L\|=L(1)=1$. Hence $a\left(x_{0}\right)=\int a d \nu(a \in A)$ for a measure $\nu$ such that supp $\nu \subset F_{0}, \nu \geqq 0$ and $\|\nu\|=1$. Since $F_{0}$ is a peak set for $\operatorname{Re} A$, there is an $f_{0} \in A$ such that $\operatorname{Re} f_{0}=1$ on $F_{0}$ and $\left|\operatorname{Re} f_{0}(x)\right|<1 \quad\left(x \in X \backslash F_{0}\right)$. Hence $1=\int \operatorname{Re} f_{0} d \nu=$ $\operatorname{Re} f_{0}\left(x_{0}\right)=\left|\operatorname{Re} f_{0}\left(x_{0}\right)\right|<1$. From this contradiction it follows that $\hat{F}_{0}=F_{0}$. The facts above show that for any $\mu \in A^{\perp}, \operatorname{supp} \mu \subset F_{0}$ and $\mu_{F_{0}}=\mu \in A^{\perp}$. It implies (ii).

(ii) $\rightarrow$ (iii). By Lemma 2.1, $A$ is self-adjoint. It implies that $A=$ $\operatorname{Re} A \oplus i \operatorname{Re} A$. From this, $\operatorname{Re} A=A \cap C_{R}(X)$ and $\operatorname{Re} A$ is closed in $C_{R}(X)$. Now, a set which is both a peak set and a BEP-set for a function space $B$ is always a sharp peak set for $B$, that is, for each closed subset $G$ in $X$ disjoint from $F$ and for each $\varepsilon>0$ there is an $f \in B$ such that $f=1$ on $F,|f|<1$ elsewhere and $|f| \leqq \varepsilon$ on $G$ (cf. [5]).

From the facts stated above, $B=\operatorname{Re} A\left(=A \cap C_{R}(X)\right)$ is a real function space on $X$ and every peak set for $B$ is a sharp peak set for $B$. Hence $\operatorname{Re} A=B=C_{R}(X)$ ([2], Theorem 5). It follows that $A=C_{R}(X) \bigoplus i C_{R}(X)=$ $C(X)$.

(iii) $\rightarrow$ (iv) and (iv) $\rightarrow$ (i) are clear.

EXAMPLE. In connection with Theorem 2.2, we here give an example of function spaces $A(\neq C(X))$ which have the properties " $F$ is an NPEPset for $A$ for any peak set $F$ for $\operatorname{Re} A$ " and " $F$ is a peak set for $A$ whenever $F$ is a peak set for $\operatorname{Re} A$ ".

Let $A=\left\{f \in C([0,1]): f(0)=\int_{0}^{1} f(t) d t\right\}$. Then $A$ is a function space on $X=[0,1]$. We easily see that a real function $g$ in $C([0,1])$ belongs to $\operatorname{Re} A$ if and only if $g(0)=\int_{0}^{1} g(t) d t$. From this, the following properties for a closed subset $F_{0}$ in $X$ are equivalent:

(1) $F_{0}$ is a peak set for $\operatorname{Re} A$, (2) $F_{0}$ is a peak set for $A$ and (3) $F_{0}=X$ or $F_{0} \nexists 0$.

From this, we see that any peak set $F_{0}$ for $\operatorname{Re} A$ is an NPEP-set for $A$. And if $F_{0}$ is a peak set for $\operatorname{Re} A$, then $F_{0}$ is a peak set for $A$. But $A$ does not coincide with $C(X)$. 


\section{References}

[1] L. Asimow and A.J. Ellis, Convexity and Its Applications in Functional Analysis, London, Math. Soc. Monographs, No. 16, Academic Press, New York, 1980.

[2] E. BRIEM, A characterization of simplexes by parallel faces, Bull. London Math. Soc., 12 (1980), 55-59.

[ 3 ] E. BRIEM, Bounded extentions and characterization of $C(X)$, Math. Z., 197 (1981), 421427.

[4] E. Briem, Peak sets for the real part of a function algebra, Proc. Amer. Math. Soc., 85 (1982), 77-78.

[5] P. C. CuRTis, Topics in Banach Spaces of Continuous Functions, Lecture Notes Series, No. 25. Aarhus University, 1970.

[6 ] T. W. Gamelin, Restrictions of subspaces of $C(X)$, Trans. Amer. Math. Soc., 113 (1964), 278-286.

[7] I. GlicksBerg, Function algebras with closed restrictions, Proc. Amer. Math. Soc., 14 (1963), 158-161.

[8] G. M. LEIBowitz, Lectures on Complex Function Algebras, Scott Foresman and Company Glenview, Ill., 1970.

[9] Junzo WADA, On the Šilov boundaries of function algebras, Proc. Japan Acad., 39 (1963), 425-428.

Present Address:

DEPARTMENT OF MATHEMATICS

ScHOOL OF EDUCATION

WASEDA UNIVERSITY

Nishiwaseda, Shinjuru-ru, Tokyo, 160 